\title{
Turbulent Buoyant Flow and Pressure Variations around an Aircraft Fuselage in a Cross Wind near the Ground
}

\author{
H. S. KOU and K. T. YANG \\ Department of Aerospace and Mechanical Engineering \\ University of Notre Dame \\ Notre Dame, Indiana 46556, USA
}

\section{J. R. LLOYD}

Department of Mechanical Engineering

Michigan State University

East Lansing, Michigan 48824, USA

\section{ABSTRACT}

Two-dimensional numerical finite-difference calculations have been carried out to study the effects of cross wind speeds and the elevation of the fuselage on turbulent buoyant flow and pressure variations around an aircraft fuselage engulfed in a simulated fire in a uniform cross wind near the ground. Detailed velocity, temperature, smoke concentration, and pressure fields have been obtained and it is found that a major influence on the physical phenomena is the relative strength of the cross flow and the buoyant flow.

\section{INTRODUCTION}

In survivable aircraft accidents involving fires, there is a critical need to understand and predict flow of hot toxic gases inside the aircraft cabins. While the fire may be initiated inside the cabin, there is an equally important accident scenario in which a fire is initiated outside the fuselage due to fuel spill and spreads into the cabin through an opening (Emmons, 1982). In the latter scenario, the fire spread into the cabin is dictated by the flow and pressure fields surrounding the fuselage, which in turn depend on the location and strength of the fire source, the elevation of the fuselage, and the speed of the prevailing wind. The development of appropriate predictive schemes for such a scenario can ultimately provide needed inputs to the eventual development of fire-safety countermeasures. The purpose of this paper is to present some results of a recent numerical study addressing this very scenario to determine the physical effects of the various parameters on the flow and pressure fields surrounding the fuselage (Kou, 1984).

Similar to an earlier companion study dealing with fire and smoke spread inside aircraft cabins (Yang et al, 1984), the numerical computations are based on a two-dimensional, primitive-variable, differential field model which includes the effects of strong buoyancy, turbulence and compressibility. Flow, temperature, and smoke concentration fields are all calculated. An algebraic turbulence model which accommodates both local and stratification effects has been utilized. The fire located outside the fuselage, which is approximated by a circular cylinder, is simulated by a volumetric heat and smoke source with arbitrarily prescribed flame envelope and rates of local heat and smoke

H. S. Kou is currently at the Tatung Institute of Technology, Taipei, Taiwan, Republic of China. 
generation. Calculations have been carried out for a fixed fire and smoke source which simulates the full-scale fire tests conducted at the Technical Center of the U. S. Federal Aviation Administration, but varying fuselage elevations and prevailing wind speeds.

\section{MATHEMATICAL FORMULATION AND NUMERICAL ANALYSIS}

Figure 1 depicts the configuration for the solution field where a cylindrical coordinate system is employed in the vicinity of the simulated fuselage, and the far region away from the fuselage is represented by a Cartesian coordinate system. The prevailing wind is at a uniform speed $U_{0}$. For the speeds considered in this study, the turbulent wall layer next to the ground is very thin, and therefore is neglected. The flame envelope on the ground, which represents the fire and smoke source, is taken to simulate a real fire, and its dimension and strengths will be described later. The Cartesian coordinate system has its origin located at the left lower corner of the solution field. The origin of the cylindrical coordinate system is located at the center of the circular cylinder (simulated fuselage).

The conservation field equations for mass, momentum, energy and smoke concentration in terms of flux quantities are well known in both coordinate systems. For illustrative purposes, these equations in Cartesian coordinates can be non-dimensionalized by introducing the following definitions:

$$
x=\frac{\bar{X}}{\bar{D}}, Y=\frac{\bar{y}}{\bar{D}}, t=\frac{U_{0} \bar{t}}{D}, \quad U=\frac{\bar{U}}{U_{0}}, \quad v=\frac{\bar{v}}{U_{0}}, \quad T=\frac{\bar{T}}{T_{0}}
$$

$\rho=\frac{\bar{\rho}}{\rho_{0}}, \rho_{\varepsilon}=\frac{\overline{\rho_{\varepsilon}}}{\rho_{0}}, \quad P=\frac{\bar{p}-\vec{p}_{\varepsilon}}{\rho_{0} U_{0}{ }^{2}}, \quad S=\frac{\bar{S}}{S_{0}}, \quad S^{\circ}=\frac{\dot{S} D}{\rho_{0} U_{0} S_{0}}, \quad Q^{\circ}=\frac{\dot{Q} D}{\rho_{0} D_{0} D_{0} p_{0}}$

Here, the subscript o denotes reference quantities, and the subscript $\varepsilon$ represents the hydrostatic equilibrium conditions. It is noted here also that $\mathrm{S}_{0}$ is simply a convenient reference concentration. The resulting non-dimensionalized equations, under the assumptions of no chemical reaction,

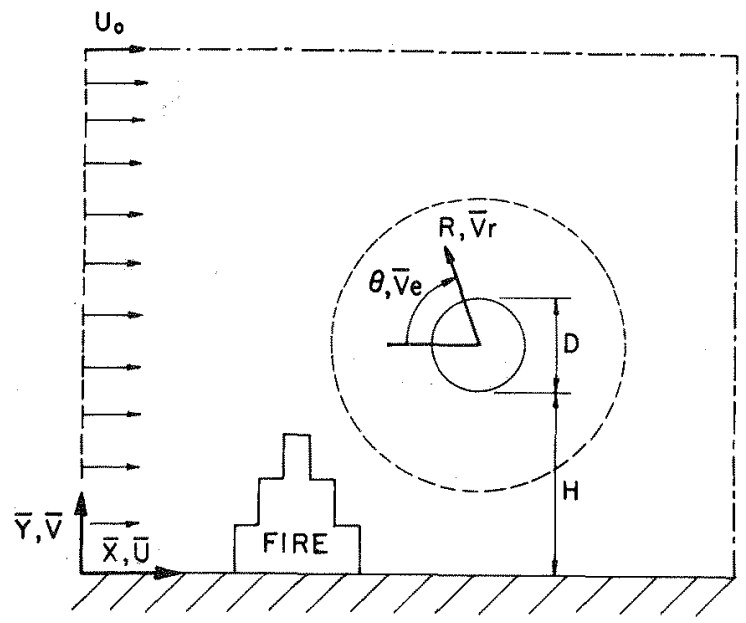

FIGURE 1. Coodinate System 
negligible viscous dissipation and pressure work, and representations of turbulent flux quantities by means of effective (1aminar plus turbulent) transport properties, can now be written as (Yang et a1, 1984; Kou, 1984)

$\frac{\partial \rho}{\partial t}+\frac{\partial(\rho U)}{\partial X}+\frac{\partial(\rho V)}{\partial Y}=0$

$\frac{\partial(\rho U)}{\partial t}+\frac{\partial\left(p U^{2}\right)}{\partial X}+\frac{\partial(\rho U V)}{\partial Y}=\frac{\partial}{\partial Y}\left[\frac{1}{R_{e}}\left(\frac{\partial U}{\partial Y}+\frac{\partial V}{\partial X}\right)\right]$

$+\frac{\partial}{\partial X}\left(\frac{4}{3 \operatorname{Re}} \frac{\partial U}{\partial X_{e}}-\frac{2}{3 \operatorname{Re}} \frac{\partial V}{\partial Y_{e}}\right)-\frac{\partial P}{\partial X}$

$\frac{\partial(\rho V)}{\partial t}+\frac{\partial(\rho U V)}{\partial X}+\frac{\partial\left(\rho V^{2}\right)}{\partial Y}=\frac{\partial}{\partial X}\left[\frac{1}{R_{e}}\left(\frac{\partial U}{\partial Y}+\frac{\partial V}{\partial X}\right)\right]$

$+\frac{\partial}{\partial Y}\left(\frac{4}{3 R_{e}} \frac{\partial V}{\partial Y}-\frac{2}{3 R_{e}} \frac{\partial U}{\partial X}\right)=\frac{\partial P}{\partial Y}-\left(\rho-\rho_{\varepsilon}\right) \frac{g D}{U_{0}^{2}}$

$\frac{\partial(\rho T)}{\partial t}+\frac{\partial(\rho U T)}{\partial X}+\frac{\partial(\rho V T)}{\partial Y}=\frac{\partial}{\partial X}\left(\frac{1}{R_{e} e^{P r}} \frac{\partial T}{\partial X}\right)$

$+\frac{\partial}{\partial y}\left(\frac{1}{\operatorname{Re}_{e} \operatorname{Pr}_{e}} \frac{\partial T}{\partial Y}\right)+Q^{\circ}$

$\frac{\partial(\rho S)}{\partial t}+\frac{\partial(\rho U S)}{\partial X}+\frac{\partial(\rho V S)}{\partial Y}=\frac{\partial}{\partial X}\left(\frac{1}{\operatorname{Re}-\frac{1}{S C_{e}}} \frac{\partial S}{\partial X_{e}}\right)$

$+\frac{\partial}{\partial Y}\left(\frac{1}{\operatorname{Re}_{e} \operatorname{Pr}_{e}} \frac{\partial S}{\partial Y}\right)+S^{*}$

$\rho T=\frac{U_{0}^{2}}{R_{0} T_{0}} P+P_{\varepsilon}$

$\rho_{E}=\exp \left[-\left(\frac{\mathrm{gD}}{\mathrm{R}_{0} \mathrm{~T}_{0}}\right) \mathrm{Y}\right]$

where $\mathrm{Re}_{e}, \mathrm{Pr}_{\mathrm{e}}$, and $\mathrm{Sc}_{e}$ are the local effective Reynolds number, Prandt number, and Schmidt number, respectively, defined by

$R e_{e}=\frac{P_{0} U_{0} D}{\bar{\mu}_{e f f}}, \operatorname{Pr} r_{e}=\frac{\bar{\mu}_{e f f} C_{p}}{\bar{k}_{e f f}}, S c_{e}=\frac{\bar{\nu}_{e f f}}{\bar{D}_{e f f}}$

where $\bar{\mu}_{\text {eff }}, \bar{k}_{\text {eff }}$ and $\bar{D}_{\text {eff }}$ are the effective (1aminar plus turbulent)

viscosity, conductivity and mass diffusion coefficient, respectively. It is understood that both $Q^{\circ}$ and $S^{\circ}$ are taken to be zero outside the flame envelope.

Before the above equations can be solved, closure models for the turbulent transport properties must be introduced. In the present study, an algebraic 
turbulence model for recirculating buoyant flows with wide variations in the turbulence level, which accounts for both local shear and stratification effects, is employed (Nee and Liu, 1978). It is given in a non-dimensional form by

$\mu_{e f f}=\frac{\bar{\mu}_{e f f}}{\mu_{0}}=1+\frac{\left[\left(\frac{\partial U}{\partial Y}\right)^{2}+\left(\frac{\partial V}{\partial X}\right)^{2}\right]^{1 / 2}\left(\frac{l}{D}\right)^{2}}{2+\frac{R j}{P_{t}}}$

where $\mu_{0}$ is the reference molecular viscosity and $\ell$ is a mixing length given by

$\frac{\ell}{D}=K\left\{\frac{\left(U^{2}+V^{2}\right)^{1 / 2}}{\left[\left(\frac{\partial U}{\partial X}\right)^{2}+\left(\frac{\partial U}{\partial Y}\right)^{2}+\left(\frac{\partial V}{\partial X}\right)^{2}+\left(\frac{\partial V}{\partial Y}\right)^{2}\right]^{1 / 2}}\right.$

$\left.+\frac{\left[\left(\frac{\partial U}{\partial X}\right)^{2}+\left(\frac{\partial U}{\partial Y}\right)^{2}+\left(\frac{\partial V}{\partial X}\right)^{2}+\left(\frac{\partial V}{\partial Y}\right)^{2}\right]^{1 / 2}}{\left[\left(\frac{\partial^{2} U}{\partial X^{2}}\right)^{2}+\left(\frac{\partial^{2} U}{\partial Y^{2}}\right)^{2}+\left(\frac{\partial^{2} V}{\partial X^{2}}\right)^{2}+\left(\frac{\partial^{2} V}{\partial Y^{2}}\right)^{2}\right]^{1 / 2}}\right]$

where $K$ is an adjustable constant and $\mathrm{Ri}$ is the gradient Richardson number given by

$R i=\frac{g D\left(\frac{\partial T}{\partial Y}\right)}{U_{0}^{2}\left(\frac{\partial U}{\partial Y}\right)^{2}}$

Furthermore, the effective conductivity $\bar{k}_{e f f}$ and effective mass diffusion coefficient $\bar{D}_{\text {eff }}$ are related to the effective viscosity $\bar{\mu}_{e f f}$ by

$\frac{1}{P_{e}}=\frac{\bar{k}_{e f f}}{\bar{\mu}_{0} C_{p}}=\frac{1}{P r}+\frac{1}{P_{t}}\left(\mu_{e f f}-1\right)$

$\frac{1}{S c_{e}}=\frac{\rho_{0} \bar{D}_{\text {eff }}}{\vec{\mu}_{0}}=\frac{1}{S c}+\frac{1}{S c_{t}}\left(\mu_{e f f}-1\right)$

where $\mathrm{Pr}$ and Sc are the molecular Prandt and Schmidt numbers, respectively. In the present study, both the turbulent Prandtl number Prt and the turbulent Schmidt number Sct are taken to be unity.

The governing equations (2) through (8) and the turbulence model given in Eas. (10) through (12) can be similarly written for the cylindrical coordinate system by noting that

$u=-v_{r} \cos \theta+v_{\theta} \sin \theta$
$v=v_{r} \sin \theta+v_{\theta} \cos \theta$

and the corresponding coordinate relations as represented in Figure 1 , and they have been given by Kou (1984). For the sake of conserving space, they will not be repeated here. The geometrical linking of the two coordinate systems will be described later. The boundary conditions are easily prescribed. The velocity components on the ground and around the cylinder surface are zero, and the velocity $U$ along $X=0$ is uniform at $U_{0}$. Theoretically, the solution reqions are infinitely extended in both positive $X$ and $Y$ directions. The heat and smoke source located inside the flame envelope provides the driving force for the 
temperature and smoke concentration fields. The ground and the cylinder (fuselage) surface are taken to be thermally insulated, and at the same time there is no net smoke particle deposition there. Temperatures and smoke concentrations away from the heat source including those along $X=0$ are taken to be ambient at $T_{0}$ and $S=0$, respectively.

In the present study, the governing equations, subjected to the boundary conditions described above, have been solved numerically by finite differences based on a micro-control volume scheme with primitive variables originally introduced by Patankar and Spalding (1972) in a manner similar to that used in an earlier companion study dealing with aircraft cabin fires (Yang et al, 1984). However, the formulations of the finite-difference equations and the associated calculation algorithm are more complicated due to the hybrid (rectangular and cylindrical) grid system used. Details of the numerical scheme are given by Kou (1984) and also contained in a companion paper (Kou, Yang and Lloyd, 1985), together with the results of validation studies based on this numerical scheme. It suffices here to describe briefly several of the salient features of the numerical computations.

A typical calculation grid system for $H / D=1.0$ is shown in Figure 2. It is seen that the circular cylinder is deliberately placed close to the left lower corner of the computational grid to accommodate the extent of the thermal piume in the region above the fire and shedding of the flow behind the cylinder. The boundary conditions at the top and right free boundaries are prescribed as follows: The gradients for both velocity components norma 1 to the boundary are taken to be zero. Also at any point along either of the free boundaries, the gradients of temperature and smoke concentration are also taken to be zero if the flow is outward, and when the flow is inward, ambient values of $T$ and $S$, i.e. $T=1$ and $S=0$ are specified at that point. These are sometimes known as the naturat boundary conditions.

One unique feature of the grid system used here is the hybrid mesh depicted in Figure 2. While the computations in either the rectangular grid or the cylindrical grid can be carried out in a standard manner, overlapping interfacial cell regions are provided to allow continuation of calculations from one region into the other. Even though the present study is only concerned with long-time behaviors, calculations marching in time are carried out until the long-time solution is achieved. Within each time step, finite-difference solutions for the two coordinate regions are obtained alternately until

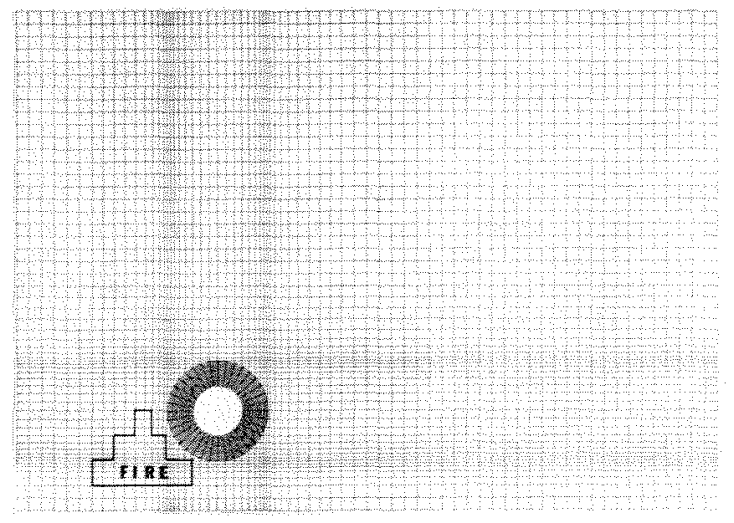

FIGURE 2. The Grid for the Case of Height Ratio $H / D=1.0$. 
convergence is reached. Calculations for each region are based on the conditions at the interface cells, and these values can be transferred from one region to the other by using standard bilinear interpolation formulae. Within each coordinate region, the computations proceed in a manner identical to that described by Yang et al (1984) and hence will not be repeated here. It is however important to note that the procedure does utilize upwind differencing for the convective terms and hence numerical errors due to false diffusion can be expected at large cell Peclet numbers (Patankar, 1980). However, due to relatively large effective viscosities encountered in the present problem, the cell Peclet number is found to be reasonably sma 11, indicating that the effect of false diffusion should also be small.

The validity of the entire calculation procedure, including the part that deals with iterations involving both coordinate regions, has been established by a series of validation studies. Results of these studies have been given by Kou (1984), and are also included in a paper describing the details of the numerical procedure (Kou, Yang and Lloyd, 1985).

As will be described later, results for the buoyancy-dominated situations all show long-time oscillatory behaviors in the flow field. A question rises then as to whether such oscillatory behaviors are physically real. There are pervasive reasons that they are indeed real. The full-scale tests at the Technical Center of the U.S. Federal Aviation Administration also showed large-scale oscillations (EkTund, 1981). In preliminary calculations utilizing much coarser grid systems, such oscilltions have also been found (Kou, 1984). Even though no three-dimensional calculations have been made, there is evidence that the oscillatory flow field is predicted by both two-dimensional and three-dimensional calculations (Satoh et a1, 1983). The resulting oscillatory frequencies are very similar, while the amplitudes for the three-dimensional cases are smaller. This is expected in view of the fact that two-dimensional flows are generally more stiff. Finally, it has also been found that the Richardson number in the turbulence model, equation (12), is important and affects both the frequency and amplitudes of the oscillations.

\section{RESULTS OF SIMULATION STUDIES}

The numerical procedure mentioned above is utilized in a series of simulation studies to determine the combined effects of the buoyant flow due to the fire and smoke source and the forced flow due to the prevailing wind speed on the velocity, temperature and smoke concentration fields in the neighborhood of the fuselage as a function of the wind speed and the elevation of the fuselage from the ground. Of particular interest is the resulting pressure variations, and smoke concentrations around the fuselage, since these are the physical parameters that will determine the fire and smoke hazards inside the aircraft cabin, if there is an opening present in the fuselage as a result of a survivable aircaft accident.

With reference to Figure 2, the diameter of the fuselage is taken to be 3.05 meters (10 feet), and the corresponding calculation doma in is given by $x=13$ and $Y=9$. The center of the fuselage is located at $X=3.5$ from the left free boundary. The depth of the field is arbitrarily taken to be 6.10 meters (20 feet) and is only used to determine the strengths of the fire and smoke source in accordance with the ful1-scale tests performed at the Technical Center of the U.S. Federal Aviation Administration. As a result, the fire source is taken to be at a constant strength and has a total power output of $75,887 \mathrm{kw}$ and a tota 1 rate of smoke generation of $0.1314 \mathrm{~kg} / \mathrm{sec}$, corresponding to that for a very 
dirty fire. The location of the fire is taken to be fixed, and the fire envelope shape is also fixed as shown in Figure 2 to approximate that for a real fire. This approximation may indeed not be too realistic, especially in cases where the forced flow is strong. However, since the effect of the cross flow on the flame slape is not a priori known, it is difficult to model. This can only be remedied by introducing a combustion model in the calculations. Simulation studies have been carried out for fuselage elevations of $H / D=0.5,1.0$ and 1.5 and wind speeds of $3.05 \mathrm{~m} / \mathrm{sec}(10 \mathrm{ft} / \mathrm{sec}), 6.10 \mathrm{~m} / \mathrm{sec}(20 \mathrm{ft} / \mathrm{sec})$ and $9.14 \mathrm{~m} / \mathrm{sec}$ $(30 \mathrm{ft} / \mathrm{sec})$. Calculations have alseo been carried out for the case of zero wind speed corresponding to pure natural convection or fire plume situations and the case of zero heat input which corresponds to pure forced flow over a circular cylinder near a ground. Detailed results have been given by Kou (1984). Since the limited space here does not permit their full presentation, only selected results are given in the following:

Before these results are presented, however, it is expedient here to make several general observations to facilitate the physical interpretation later on. Firstly with the prescribed heat source, the range of wind speeds covered in this study does span the complete region of interest ranging from forced-flow dominated situations $(9.14 \mathrm{~m} / \mathrm{sec})$ to natural-convection dominated flow $(3.05$ $\mathrm{m} / \mathrm{sec}$ ). Secondly, forced flow tends to stabilize the flow, while the buoyancy effect due to the fire source destabilizes it. Thirdly, strong buoyancy effects lead to long-time behaviors which are oscillatory, while time-independent steady-state behaviors are obtained when forced-flow effects are dominant. Fourthly, for elevation parameters H/D exceeding unity, the ground effect is not significant. For H/D less than unity, the reduction in local flow area and the ground friction become increasingly important and generally have the opposite effects on the flow field. Lastiy, in view of the similarity of the governing equations and the boundary conditions, results for the temperature field are very similar to that for the smoke concentration field. Consequentiy, separate physical interpretations of these two fields are not necessary.

Figures $3(\mathrm{a})$ and (b) are typical smoke concentration contour plots for the case of $H / D=0.5$. Figure $3(\mathrm{a})$ is for a wind speed of $3.05 \mathrm{~m} / \mathrm{sec}(10 \mathrm{ft} / \mathrm{sec})$ and Figure $3(\mathrm{~b})$, for the speed of $6.10 \mathrm{~m} / \mathrm{sec}(20 \mathrm{ft} / \mathrm{sec})$. In both cases, buoyancy effects are significant and long-time behaviors are oscillatory due essentially to the instability of the fire plumes. The absolute time instants noted in these figures have no special meaning, other than that reflecting the period duing the oscillations. At the lower speed in Figure 3(a), the effect of the wind on the fire plume is still minimal, and the fuselage is not much affected by the fire. The temperatures on the surface are essentially ambient. When the wind speed is doubled (Figure 3(b)), the fire plume is swept in the direction of the wind and the fuselage is largely engulfed by the fire. This represents the situation where both forced flow and natural convection play equaliy important roles. As already noted previously, similar oscillatory behaviors of the smoke field have been observed in the full-scale tests at the Technical center of the U. S. Federal Aviation Administration (Eklund, 1981). Unfortunately no direct comparisons can be made because of the qualitative nature of the tests.

One important result in the present study, as pointed out previously, is the pressure variation around the fuselage. Figure 4 describes such pressure variations at $\theta=0^{\circ}$ and $180^{\circ}$ for $H / D=0.5$ and a wind speed of $9.14 \mathrm{~m} / \mathrm{sec}(30$ $\mathrm{ft} / \mathrm{sec}$ ). It is seen that the buoyancy effect induces a regular oscillation in the long-time behavior and the period is about 4 seconds. Additionally, it has been found that the pressure depressions at the bottom of the fuselage are higher than that at the top. It is believed that the buoyancy in the fire 

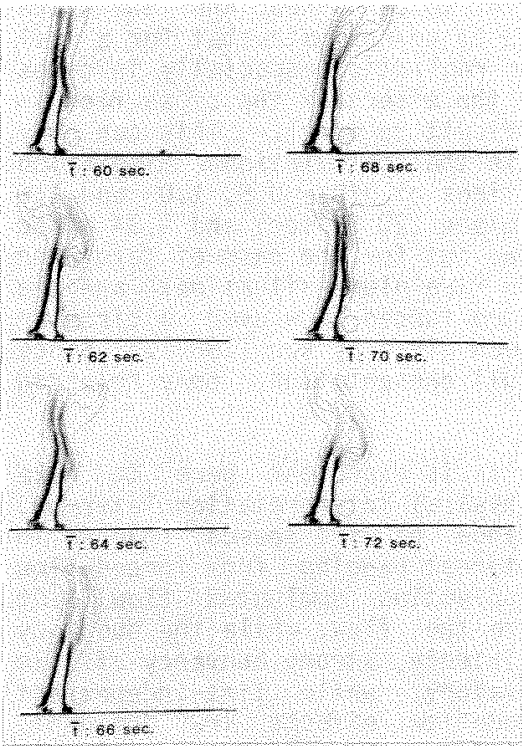

(a) $U_{0}=3.05 \mathrm{~m} / \mathrm{sec}$

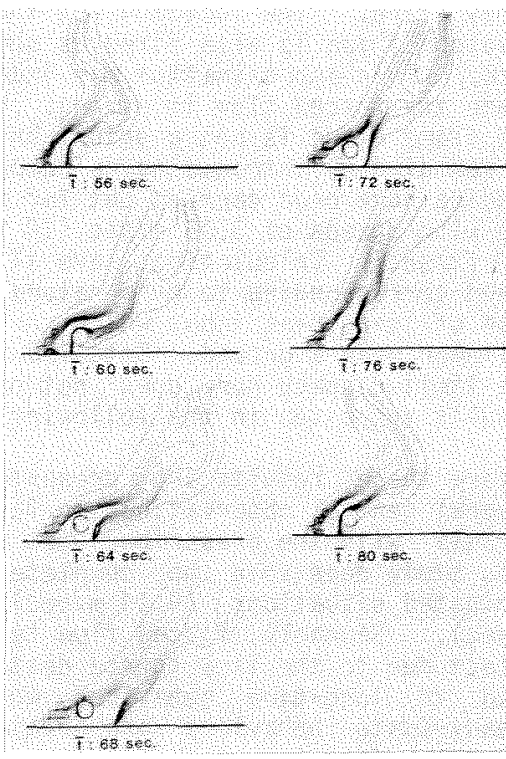

(b) $U_{0}=6.10 \mathrm{~m} / \mathrm{sec}$

FIGURE 3. Oscillatory Smoke Concentration Fields for $H / D=0.5$.

accelerates the flow more in the region below the fuselage, causing the pressure to decrease there, despite the slight drag offered by the presence of the ground. Figure 5 shows the effect of reducing the wind speed from $9.14 \mathrm{~m} / \mathrm{sec}$ $(30 \mathrm{ft} / \mathrm{sec})$ to $6.10 \mathrm{~m} / \mathrm{sec}(20 \mathrm{ft} / \mathrm{sec})$ at the same fuselage elevation $\mathrm{H} / 0=0.5$. The increased buoyancy effect in this case further destabilizes the flow and the long-time oscillatory behavior is no longer very regular. The presence of the ground seems to damp out some of the oscillations in the region below the

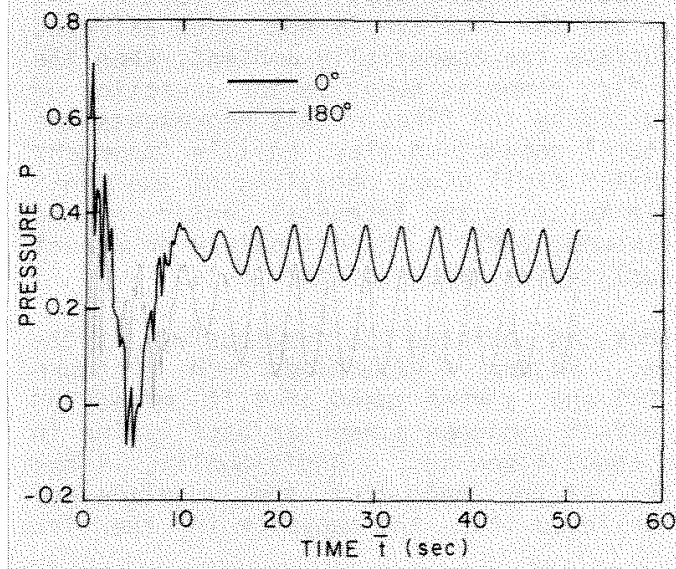

FIGURE 4. Pressure at $\theta=0^{\circ}$ and $180^{\circ}$ for $U_{0}=9.14 \mathrm{~m} / \mathrm{sec}$ and $H / D=0.5$ (Heat on). 


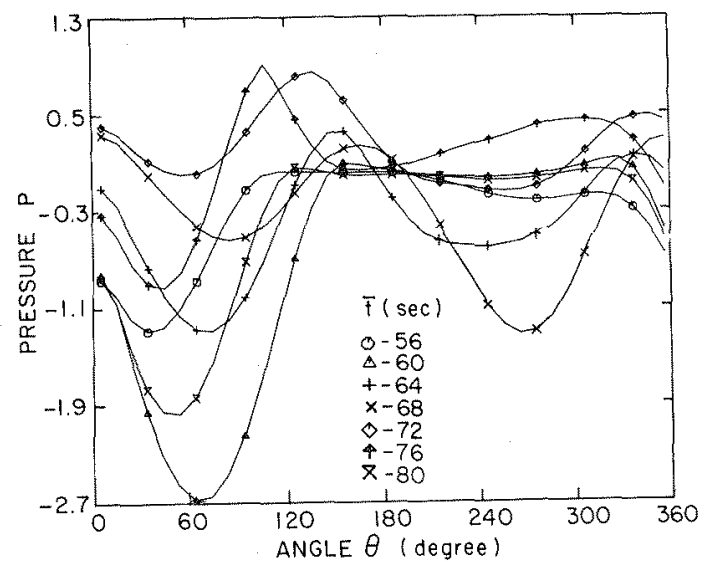

FIGURE 5. Pressure Variation Around Fuselage for $\mathrm{II}_{0}=6.10$ $\mathrm{m} / \mathrm{sec}$ and $H / D=0.5$ (Heat Source $0 n$ ).

fuselage. From Figure 5 and Figure $3(b)$, which depict the corresponding field behaviors, the period of oscillation can be approximately estimated at 24 seconds, and a more exact value can only be determined by performing a Fourier analysis of the irregular oscillations. Figure 6 shows the corresponding pressure variations for a case similar to that in Figure 8 except that the fuselage elevation is now increased to $H / D=1.5$ Somewhat irregular oscillations are still present, but the behavior at the bottom and at the top of the fuselage are no longer that different, again attesting to the fact that for this value of $\mathrm{H} / \mathrm{D}$ the ground loses much of its effect on the flow field. For this case, an approximate period is estimated at 28 seconds.

Figure 7 gives an illustration concerning the temperature variations around the fuselage, and it is understood that this same information can be interpreted as the smoke concentration variations. However, it should also be understood that $T=1$ would then correspond to $S=0$. Figure 10 is for the case of $H / D=0.5$ and a wind speed of $6.10 \mathrm{~m} / \mathrm{sec}(20 \mathrm{ft} / \mathrm{sec})$. Here the wind speed sweeps the fire toward the fuselage, more under it than above. As a result, the temperatures are higher at the lower surface of the fuselage. It is also seen that during certain times in the cycle the fire plume actually stays away from the fuselage,

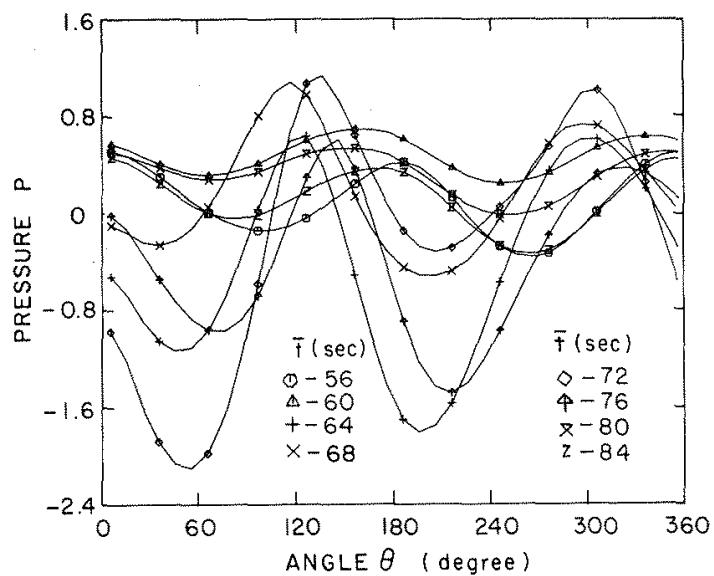

FIGURE 6. Pressure Variations Around Fuselage for $U_{0}=6.10$ $\mathrm{m} / \mathrm{sec}$ and $H / D=1.5$ (Heat Source On). 


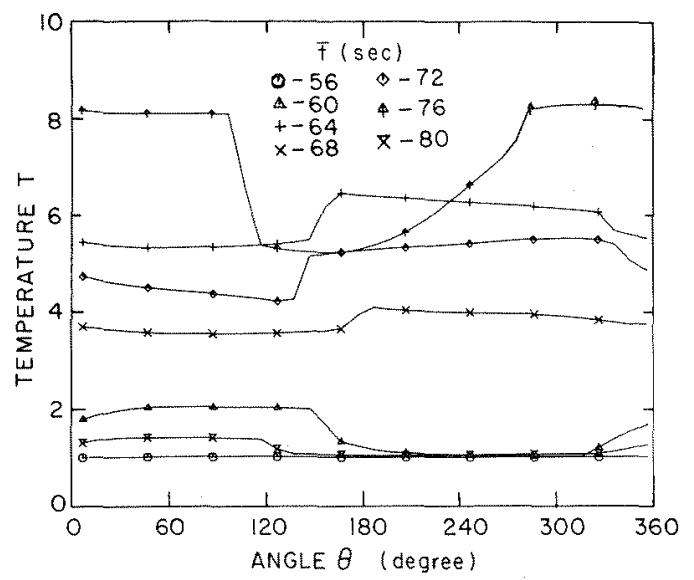

FIGURE 7. Temperature Variation Around Fuselage for $U_{0}=6.10$ $\mathrm{m} / \mathrm{sec}$ and $\mathrm{H} / \mathrm{D}=0.5$ (Heat Source on).

thus leaving the entire fuselage surface at essentially the ambient temperature. Also, during the other parts of the cycle, the surface temperature can become very high, especially at the front of the fuselage.

\section{CONCLUDING REMARKS}

A numerical study based on two-dimensional finite-difference calculations with a hybrid grid system has been carried out to study the effects of wind speed and elevation of an aircraft fuselage located downwind of a fire and smoke source on the pressure, temperature and smoke concentration variations at the surface of the fuselage. The following general conclusions can be drawn:

(a) The surface variations in pressure, temperature and smoke at the fuselage are very sensitive to the relative effects of the prevailing wind speed and the buoyant flow from the fire source. The buoyancy arises from the fire source destabilizes the flow, and causes the long-time behavior to become oscillatory. When the buoyancy effect is large, the oscillations can become rather irregular.

(b) For elevation parameters exceeding $H / D=1.0$, the ground does not exert much effect on the physical characteristics in the immediate neighborhood of the fuselage. As $H / D$ falls below unity, the ground effect becomes increasingly more important, especially when the fire is present.

The present study can only be considered as a first step in addressing the external fire problem in survivable aircraft accidents. In the reat scenario, the flow field is necessarily three-dimensional, and there is always thermal radiation exchange between the fire, the smoke, the ground and the fuselage. The combustion process involving the spilled fuel is also present in the real phenomena. Systematic studies, both numerical and experimental, are needed to address these realistic effects so that the all important fire safety issues involved in survivable aircraft accident can be rationally dealt with.

\section{NOMENCLATURE}

$\begin{array}{ll}C_{p} & \text { isobaric heat capacity } \\ D & \text { cylinder diameter } \\ D & \text { mass diffusion coefficienbt } \\ g & \text { gravitational acceleration }\end{array}$




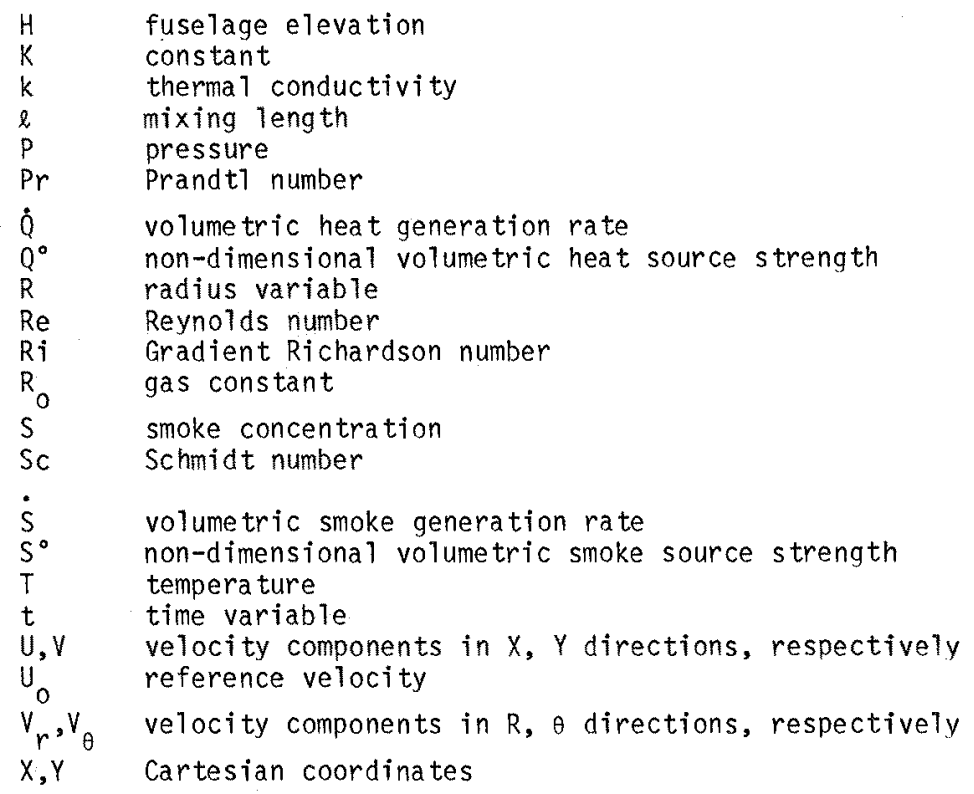

Greek Symbols

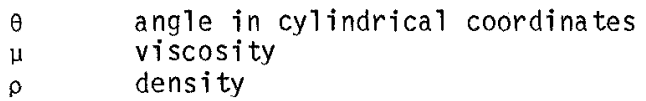

Subscripts
e based on effective transport properties
eff effective (molecular plus turbulent)
- reference quantities
$t \quad$ turbulent
$\varepsilon \quad$ hydrostatic equilibrium condition

Superscript

dimensional quantities

\section{ACKNOWLEDGEMENT}

The authors gratefully acknowledge support of the research under $U$. S. Nationa 1 Bureau of Standards Grant NB81NADA 2000 through an interagency aqreement with the Federal Aviation Administration. 


\section{REFERENCES}

Eklund, T. (1981). personal communication.

Emmons, H. W. (1982). The ingestion of flames and fire gases into a hole in an aircraft cabin for arbitrary tilt angles and wind speed. Home Fire Project TR No. 52, Division of Applied Sciences, Harvard University, Cambridge, MA.

Kou, H. S. (1984). Turbulent buoyant flow and pressure variations around a circular cylinder in a cross uniform flow near the ground. Ph.D. dissertation, University of Notre Dame, USA, 233 $\mathrm{pp}$.

Kou, H. S., Yang, K. T., and Lloyd, J. R. (1985). A numerical study of mixed convection for flow over a circular cylinder near a ground in the presence of a volumetric heat source. Paper under review for publication.

Nee, V. W. and Liu, V. K. (1978). An algebraic turbulence model for buoyant recirculating flow. Technical Report TR-79002-78-2, Dept. of Aerospace and Mechanical Engineering, University of Notre Dame, $107 \mathrm{pp}$.

Patankar, S. V. (1980). Numerical Heat Transfer and Fluid Flow, McGraw Hill Book Co.

Patankar, S. V. and Spalding, D. B. (1972). A calculation procedure for heat, mass and momentum transfer in three-dimensional parabolic flow, Int. J. of Heat and Mass Transfer, vol. 15, 1787.

Satoh, K., Yang, K. T., Lloyd, J. R. and Kanury, A. M. (1983). A Numerical Finite-Difference Study of the Oscillatory Behavior of Vertically Vented Compartments, Numerical Properties and Me thodologies in Heat Transfer, Proc. 2nd Nat. Sym., ed. T. M. Shih, Hemisphere publishing Corp., 517.

Yang, K. T., Lloyd, J. R., Kanury, A. M. and Satoh, K. (1984). Modeling of turbulent buoyant flows in aircraft cabins, Comb. Sci. and Tech., vol. 39, 107. 